\title{
Preface of the Special Issue Quantum Foundations: Theory and Experiment
}

\author{
Andrei Khrennikov • Gregor Weihs
}

Received: 23 February 2012 / Accepted: 10 March 2012 / Published online: 24 March 2012

(C) Springer Science+Business Media, LLC 2012

The present wave of interest in quantum foundations is caused by the tremendous development of quantum information science and its applications to quantum computing and quantum communication. Nowadays this interest even increases, because it became clear that some of the difficulties encountered in realizations of quantum information processing are not simply technicalities, but instead have roots at the very fundamental level. To solve such difficult problems, theory has to keep up with experiment. This issue presents theoretical and experimental viewpoints on a number of foundational problems having direct relations to quantum information.

Born's Rule Can it be violated? In the framework of conventional quantum mechanics (QM), e.g. with the Copenhagen interpretation, this question is considered as ill-posed. Max Born simply postulated this rule in a footnote in his paper [1]. If it could be derived, one could reduce the number of axioms of QM. Therefore many attempts to derive this rule can be found in the literature on quantum foundations, either from within the remaining axioms of QM and general physical considerations [3] or from another set of perhaps "more basic" postulates, or finally by going beyond QM, i.e. considering subquantum models. So far, there is no generally accepted derivation of the Born rule, but this does not imply that such a derivation is impossible [2]. Among recent attempts to derive Born's rule from theories with hidden variables, we can mention the work of G. 't Hooft [4], which is devoted to a deterministic subquantum model. He claims that the quantum calculus of probabilities based

\footnotetext{
A. Khrennikov (凶)

International Center for Mathematical Modelling in Physics and Cognitive Sciences, Linnaeus University, Växjö 35195, Sweden

e-mail: andrei.khrennikov@lnu.se

G. Weihs

Institut für Experimentalphysik, Universität Innsbruck, Technikerstraße 25, 6020 Innsbruck, Austria
} 
on squaring of complex probability amplitudes and the linear dynamics for these amplitudes can be derived from purely deterministic classical mechanics. Another recent attempt to derive Born's rule and also to find roots of its possible violation using a prequantum classical statistical field theory (PCSFT) is presented in the paper of this issue "Towards a Field Model of Prequantum Reality" by A. Khrennikov, doi:10.1007/s10701-011-9611-y. This theory is essentially a modern presentation of Schrödinger's undulatory mechanics as a theory of random fields. In PCSFT, Born's rule can be derived as an approximate rule for the calculation of quantum probabilities, with the main term corresponding to integration of the squared prequantum field. However, in PCSFT detectors can also integrate higher order field nonlinearities. This approach and t'Hooft's approach are both about the reduction of QM to a classical model.

On the other hand, many years ago R. Sorkin contemplated the question of whether QM departs sufficiently from the laws of classical Kolmogorovian probability. He elaborated a series of nonclassical models of probability that are even more exotic compared to classical probability than conventional QM. In particular, Born's rule could be violated. Sorkin's violation of Born's rule may not be visible in the two slit experiment, but instead in a triple slit experiment. Such a test is described by I. Söllner et al. in the paper of this issue "Testing Born's Rule in Quantum Mechanics for Three Mutually Exclusive Events”, doi:10.1007/s10701-011-9597-5. A particular focus is given to distinguishing technical nonlinearities in the experiment from the nonlinearity of a hypothetical violation of Born's rule. The authors are able to put a much tighter bound on such a possible violation of Born's rule than previously possible. We remark that the problem of a violation of Born's rule is not only of fundamental foundational interest, but it would also enable "super-quantum computing". The question on possible generalizations of QM in the spirit of Sorkin's proposal are also being studied in the paper of this issue "Generalized Quantum Probability and Entanglement Enhancement Witnessing" by G. Jaeger who extends Sorkin type deviations from QM and their experimental investigation to the context of two-particle interference measurements. In particular, it is suggested that deviations from Born's rule may be amplified in two-particle interference due to entanglement. Furthermore, these suggested experiments are also sensitive to other possible deviations of the interference of microscopic systems from the predictions of standard QM.

Contextuality Recently the contextuality of QM has attracted a lot of interest. The question whether QM is a nonlocal theory with hidden variables such as e.g. Bohmian mechanics or whether even nonlocality cannot explain the complexity of quantum statistical data is a hot topic of current debates on the foundations of QM. Contextuality as the basic feature of quantum measurements was emphasized already by N. Bohr, then it was made manifest in the Kochen-Specker theorem, and recently it was tested experimentally by using Bell-type inequalities for compatible observables of non-compound quantum systems. One important consequence of these studies of contextuality of quantum measurements is a better understanding of the essence of entanglement: as entanglement of observables (in particular, for a single quantum system) and not of the systems themselves. The group of $\mathrm{H}$. Rauch performed interesting experimental studies of contextuality in the framework of neutron interferometry. An 
extended review on this field can be found in the paper of this issue "Neutron Matter Wave Quantum Optics", doi:10.1007/s10701-011-9569-9. In addition to general aspects recent experiments are discussed that demonstrated entanglement of internal (spin) and spatial (pathway) degrees of freedom of neutron and measurements of the geometric phases.

Quantum Foundations and High Energy Physics Quantum mechanics has existed for more than 100 years but some long-standing debates are still alive and waiting for a solution. In a different field, in particle physics, experiments are achieving ever higher precision such that the borderline of known physics will be reached. Combining both fields and aiming to tackle some of the most fundamental questions of quantum mechanics and to relate them to the most fundamental questions in particle physics seems obvious. The contribution "Heisenberg's Uncertainty Relation and Bell Inequalities in High Energy Physics" by A. Di Domenico et al., doi:10.1007/s10701-011-9575-y, develops a formalism that is capable to determine the information theoretic content of observables measured in accelerator facilities. Herewith, fundamental questions of quantum information theory can be addressed. For example, one can ask the question how CP violation, an asymmetry of matter and antimatter, changes the information theoretic content of the quantum system under consideration.

The formalism developed in this contribution for decaying and oscillating systems also allows for the first time to rewrite expectations values in operator form and, consequently, use standard methods from quantum information theory. In the last section of the contribution the formalism is applied to Bell inequalities for entangled K-mesons. This allows a simple computation without optimization. A comparison with the uncertainty shows that these massive systems at high energy possess different nonlocal features than systems of ordinary matter and light.

Weak Measurements are another hot topic of modern theoretical and experimental studies. This issue contains the work "Experimental Evidence for a Dynamical Non-locality Induced Effect in Quantum Interference Using Weak Values", doi:10.1007/s10701-011-9596-6, of two experimenters, S.E. Spence and A.D. Parks, in which weak measurements are used to study concepts of modular momentum and dynamical non-locality. Although the non-local exchange of modular momentum associated with such phenomena cannot be directly observed, it has been suggested that effects induced by this exchange can be measured experimentally using weak measurements of pre- and post-selected ensembles of particles. This paper reports on such an optical experiment that yielded measured weak values that were consistent with the theoretical prediction of an effect induced by a non-local exchange of modular momentum.

We hope that this issue will be useful for experts working in all domains of quantum physics and quantum information theory: theoreticians, experimenters, mathematical physicists and even philosophers interested in quantum foundations.

\section{References}

1. Born, M.: Zur Quantenmechanik der Stossvorgänge. Z. Phys. 37, 863-867 (1926) 
2. Landsman, N.P.: In: Weinert, F., Hentschel, K., Greenberger, D., Falkenburg, B. (eds.) Compendium of Quantum Physics. Springer, Berlin (2008)

3. Zurek, W.H.: Environment-assisted invariance, entanglement and probabilities in quantum physics. Phys. Rev. Lett. 90(12), 120404 (2003)

4. 't Hooft, G.: The free-will postulate in quantum mechanics. Her. Russ. Acad. Sci. 81(10), 907-911 (2011). arXiv:quant-ph/0701097v1 (2007) 